ISSN: 1130-3743 - ISSN electrónico: 2386-5660

DOI: http://dx.doi.org/10.14201/teoredu2015272221244

\title{
EFECTOS DE LAS CARACTERÍSTICAS DEL PROBLEMA, CAPTACIÓN DE SU ESTRUCTURA Y USO DE ANALOGÍAS SOBRE EL ÉXITO DE LOS ESTUDIANTES DE SECUNDARIA EN LA RESOLUCIÓN DE PROBLEMAS
}

\author{
Effects of problem characteristics, capturing problem \\ structure and using analogies on high school students' \\ success on problem-solving
}

\section{Effets des caractéristiques du problème, de la capture de son structure et de l'utilisation d'analogies sur le succès des lycéens dans la résolution de problèmes}

Pedro García Gallego, Vicente SAnjosé LóPez y Joan Josep Solaz-PorTolés Universitat de València. Facultad de Magisterio. Departament de Didàctica de les Ciències Experimentals i Socials. Avgda. Tarongers, 4. 46022 Valencia (España). joan.solaz@uv.es

Fecha de recepción: marzo de 2015

Fecha de aceptación: junio de 2015

Biblid [(1130-3743) 27, 2-2015, 221-244]

RESUMEN

En este trabajo se analiza la influencia del nivel académico, de los estudios cursados, de la estructura y contexto del problema, de la captación de la estructura y del uso de analogías, en la resolución de problemas. Se ha utilizado un diseño factorial con un factor intra-sujetos y varios factores entre-sujetos, que se han seleccionado en función de la hipótesis a contrastar. Se ha administrado a 181 estudiantes de 
secundaria dos tipos de cuadernillos que contienen dos problemas y preguntas sobre la captación de la estructura y uso de analogías. Los resultados de pruebas estadísticas no paramétricas muestran que: a) El nivel académico y el tipo de estudios cursados son variables cruciales en la resolución de problemas; b) La captación de la estructura influye decisivamente para resolver los problemas, pero no el uso de analogías; y c) Los estudiantes resuelven significativamente mejor los problemas de estructura sencilla y contexto familiar.

Palabras clave: didáctica de las ciencias; resolución de problemas; contexto y estructura; analogías.

\section{SUMMARY}

This study analyses the influence of academic level, academic itinerary, word problem's context and structure, capturing problem structure, and using analogies on problem-solving. A factorial design was used with one within-subjects variable and several between-subjects variables, which have been selected depending on the hypothesis in question. Two types of booklets containing two word problems and questions about capturing problem structure and using analogies in problem solving, were administered to a total of 181 grade- 10 and grade- 12 students. The results of non-parametric statistical tests showed that: a) Academic level and academic itinerary are decisive variables in problem solving, b) Capturing problem structure is needed to solve problems, but the use of analogies is not needed, and c) Problems increase in difficulty when they have complex structure and low familiarity context.

Key words: science education; problem-solving; context and structure; analogies.

\section{SOMMAIRE}

Cet article analyse l'influence du niveau académique, des études choisies, de la structure et le contexte du problème, de la capture de la structure et de l'utilisation d'analogies dans la résolution de problèmes. Il a été utilisé un plan factoriel à une variable intra-sujets et plusieurs variables inter-sujets, qui on été choisies en fonction de l'hypothèse retenue. Lycéens (181) ont rempli deux types de livrets contenant deux problèmes et les questions sur la capture de la structure et l'utilisation d'analogies. Les résultats des tests statistiques non-paramétriques montrent que: a) Les études et le niveau académique sont des variables clés dans la résolution de problèmes, b) La capture de la structure est nécessaire pour résoudre les problèmes, mais pas l'utilisation d'analogies, et c) Les problèmes qui ont une structure simple et un contexte familial peuvent être facilement résolus par les estudiants.

Mots clés: didactique des sciences; résolution de problèmes; structure et contexte; analogies. 


\section{INTRODUCCIÓN}

En las últimas cuatro décadas se han llevado a cabo gran cantidad de estudios sobre resolución de problemas. De hecho, existe un consenso creciente en relación a los factores y procesos cognitivos implicados en la resolución de problemas (Solaz-Portolés y Sanjosé, 2008a). La literatura especializada sugiere que el éxito en la resolución de un problema determinado depende de la combinación de un buen conocimiento de la materia, del conocimiento y aplicación de estrategias cognitivas y metacognitivas, y de componentes actitudinales (Solaz-Portolés, Sanjosé y Gangoso, 2013).

La comprensión de un problema parte de la comprensión de su enunciado, usualmente un texto corto. El procesamiento de la información contenida en este texto corto demanda una gran cantidad de inferencias y la activación de conocimiento previo específico conceptual, situacional, procedimental, estratégico y esquemático (Solaz-Portolés y Sanjosé, 2008b). Los modelos interactivos de la comprensión textual sostienen que comprender un texto comporta elaborar representaciones mentales del contenido (Verhoeven y Perfetti, 2008). Así, por ejemplo, Kintsch (1998) defiende que la comprensión de un texto puede tener diferentes niveles de elaboración: a) el nivel léxico o de reconocimiento de las palabras; b) la Base del Texto o nivel semántico, constituido por los significados de las oraciones independientemente de la forma en que están escritas y de las palabras usadas; y c) el Modelo de la Situación, en el que la información semántica del texto se relaciona con el conocimiento previo y se puede aplicar a nuevas situaciones. Sin embargo, más allá de la representación de los objetos y eventos del mundo ordinario, los enunciados de problemas en ciencias y matemáticas requieren representaciones de cantidades, magnitudes, operaciones y ecuaciones. Kintsch y Greeno (1985) postularon un cuarto nivel de representación mental, específico para problemas y que llamaron Modelo del Problema, que es un nivel abstracto de representación.

Una vez elaboradas las representaciones mentales de alto nivel del problema (Modelo de la Situación y Modelo del Problema), el sujeto que resuelve el problema ha de dar sentido a la demanda explícita del problema y planificar el camino por el que puede acceder a la información pedida a partir del contenido incluido en esas representaciones mentales. Se inicia la fase de resolución del problema propiamente dicha, que comporta una navegación por el espacio del problema, el cual se podría definir como el conjunto de todos los caminos posibles para resolver el problema (Newell y Simon, 1972). Esta fase de resolución implica variables como las relacionadas con las habilidades de procesamiento del sujeto, el grado de pericia o experiencia, la capacidad para realizar con éxito el llamado proceso de traducción al lenguaje matemático, y el conocimiento matemático necesario para resolver las ecuaciones planteadas (Sanjosé, Solaz-Portolés y Valenzuela, 2009). 


\subsection{La transferencia analógica}

Cuando los estudiantes utilizan conocimientos y estrategias que han desarrollado en la resolución de problemas para resolver otro problema, que representa una situación similar a la que presentaba alguno de los problemas anteriormente resueltos, se dice que se ha producido el fenómeno de transferencia analógica (Van Lehn, 1990). Rumelhart (1989) ha sugerido que la transferencia analógica es el método principal para resolver problemas nuevos en cualquier dominio, ya sean problemas académicos o de la vida cotidiana. Sternberg (1982) llegó a afirmar que es, en realidad, el único método para resolver problemas. En la resolución de problemas mediante transferencia analógica, el sujeto ha de recuperar de su memoria ejemplos resueltos antes, y usarlos para resolver el nuevo problema (Barnett y Ceci, 2002; Goldstone y Sakamoto, 2003). De acuerdo con De la Fuente (1992), para resolver un problema por analogía se debe, en primer lugar, elaborar una representación mental del problema propuesto (problema diana). A continuación, durante el proceso de resolución, se ha de evocar (activar) un problema potencialmente análogo (problema fuente) almacenado en la memoria a largo plazo. Tras la recuperación de la representación de este problema fuente, se tiene que llevar a cabo un proceso de contrastación analógica, en el que se establecen las similitudes y diferencias entre el problema diana y el problema fuente. Por último, si la evaluación del problema fuente evocado como un análogo válido es positiva, se ha de adaptar el proceso de resolución del problema fuente para obtener una solución al problema diana.

Según algunos autores (Chi, Feltovich y Glaser, 1981; Holyoak y Koh, 1987), los problemas en ciencias y matemáticas pueden caracterizarse por dos factores: 1) su contexto (también denominado historia o superficie); y 2) su estructura. El contexto alude a la temática concreta o ámbito del mundo al que pertenecen los objetos, propiedades, estados y eventos que se describen en el enunciado. La estructura se refiere a las relaciones entre variables en el espacio del problema dadas por reglas, normas, principios o leyes, y está vinculada a las representaciones abstractas propias del Modelo del Problema. En nuestro estudio, la estructura hace referencia a la relación matemática que se da entre las variables asociadas a las entidades expuestas en el enunciado.

La analogía entre problemas puede construirse en términos de su similitud superficial (contextual) y/o estructural (Holyoak, 1984). El estudio de Faries y Reiser (1988) demuestra la participación de estructuras de conocimiento abstractas en el proceso de recuperación de problemas análogos. Estos investigadores intentaron demostrar que el proceso de recuperación de problemas análogos está fuertemente influido por elementos estructurales del problema, en contra de otros trabajos que defendieron que el mecanismo de recuperación de análogos está mediado tanto por elementos superficiales como estructurales (Holland, Holyoak, Nisbett y Thagard, 1986; Keane, 1988). En el trabajo de Kurtz y Loewestein (2007) se recogen todas las investigaciones que han puesto en evidencia que la comparación de estructuras de problemas facilita la recuperación de análogos. También se ofrece 
la correspondiente explicación: la comparación estructural favorece la abstracción del "esquema del problema», que no es sino la generalización de la estructura común de los problemas, y ésta a su vez facilita la recuperación de análogos y la resolución de problemas.

Estudios realizados con estudiantes de secundaria revelan (Gómez, SolazPortolés y Sanjosé, 2013a y b): a) la importancia de la detección de similaridades estructurales entre problemas para activar los procesos de transferencia analógica, b) la posibilidad de que las similaridades superficiales apantallen las diferencias estructurales, circunstancia que puede llevar a una resolución errónea, y c) los problemas de contextos no familiares para los estudiantes (fundamentalmente de carácter científico) conllevan dificultades en la construcción de analogías y, consecuentemente, a un bajo éxito en la resolución. Se ha constatado la extraordinaria importancia que tiene para estos estudiantes la familiaridad del contexto del problema para establecer analogías estructurales. En este sentido, se ha comprobado que el porcentaje de estudiantes que puede llegar a percibir analogías estructurales entre problemas es significamente mayor cuando los enunciados de los problemas son de alta familiaridad para el estudiante (Gómez, Solaz-Portolés y Sanjosé, 2014).

\subsection{Objetivos e hipótesis}

Hasta aquí se ha hecho una sucinta revisión de conocimientos y procesos que se suelen emplear en la resolución de problemas, así como de una estrategia muy importante que suele utilizarse en esta tarea: la transferencia analógica. También se han mencionado la estructura y el contexto como dos factores que pueden caracterizar el enunciado de un problema, y el papel decisivo que ambos desempeñan en la transferencia analógica. En el caso específico de estudiantes de secundaria, hemos visto la trascendencia que tienen en la transferencia analógica la detección de la estructura y los contextos familiares de los problemas.

En este estudio se pretende estudiar cómo afectan determinadas variables a la resolución de problemas por parte de estudiantes de educación secundaria. Para estos estudiantes, es razonable pensar que el tipo de estudios cursados a lo largo de la vida académica puede influir decisivamente en su capacidad para resolver problemas. De hecho, si se siguen estudios de ciencias en la enseñanza secundaria (ESO y Bachillerato), se resuelven más problemas que si se llevan a cabo estudios de humanidades y ciencias sociales. Haber resuelto más problemas habrá, probablemente, desarrollado más sus estrategias cognitivas y metacognitivas, cruciales en esta tarea de alto nivel cognitivo, además de haber adquirido otros conocimientos necesarios para llevar a cabo con éxito dicha tarea (Solaz-Portolés, Sanjosé y Gangoso, 2013).

En concreto, con este trabajo se quiere: 
- Estudiar cómo afectan la familiaridad del contexto del enunciado y la complejidad de la estructura (relaciones matemáticas subyacentes) de los problemas a la resolución en estos estudiantes.

- Evaluar si la captación de la estructura de los problemas y el uso de analogías son herramientas imprescindibles en los procesos de resolución de problemas.

- Analizar los efectos del nivel académico y del tipo de estudios cursados (itinerario) por los estudiantes sobre la resolución de problemas de diferente contexto y complejidad estructural.

En función de lo que se ha dicho anteriormente, y teniendo presentes los antecedentes teóricos expuestos, podemos plantear las siguientes hipótesis:

- H1.- Es lógico pensar que cuanto mayor sea la complejidad de la estructura de un problema, menor éxito de resolución obtenga. Es decir, los problemas de estructura más compleja deben mostrarse más difíciles de resolver que los de estructura simple.

- H2.- Hemos visto que la captación de la estructura de los problemas y establecer analogías son elementos muy relevantes para conseguir efectuar la transferencia analógica. De acuerdo con ello, podemos formular la hipótesis 2 del siguiente modo: la captación de la estructura de los problemas y usar analogías son variables que deben estar asociadas de modo significativo con la puntuación obtenida en la resolución de los problemas.

- H3.- Se ha indicado también la importancia del nivel académico y del itinerario cursado cuando se resuelven problemas. Consecuentemente, nuestra hipótesis 3 puede enunciarse así: cuanto mayor nivel académico tengan los estudiantes y el itinerario cursado más especializado en asignaturas de ciencias, mayor debería ser el éxito en la resolución de problemas.

- H4.- Se ha encontrado en trabajos anteriores que los enunciados de problemas que implican contextos no familiares para los estudiantes dificultan la transferencia analógica. Por tanto, nuestra hipótesis 4 afirma que, para problemas de la misma complejidad estructural, los que sean de contexto más familiar para los estudiantes deben resultar más fáciles de resolver que los de contexto no familiar.

\section{Metodología}

\subsection{Diseño experimental}

Se ha utilizado un diseño factorial con un factor intra-sujetos y varios factores entre-sujetos, que serán seleccionados en función de la hipótesis a contrastar. El factor intra-sujetos es la complejidad de la estructura del problema, con dos valores, problema de estructura fácil (de un paso o una sola regla de tres simple) o Problema 1, y problema de estructura difícil (de dos o más pasos, o de dos o más 
reglas de tres simples) o Problema 2. Los factores entre-sujetos que pueden ser considerados son: el nivel académico ( $4 .^{\circ}$ de ESO/2. ${ }^{\circ}$ de Bachillerato), tipo de estudios o itinerario cursado (ciencias/letras -humanidades y ciencias sociales-), contexto del enunciado del problema (consumo de combustible de un coche/producción industrial de un compuesto químico), captación de la estructura de los problemas (con tres valores, captación completa, captación parcial y no captación) y uso declarado de analogías (con dos valores, se declara el uso/no se declara su uso).

\subsection{Sujetos}

Han participado en la investigación 181 estudiantes de ambos sexos (114 mujeres y 67 hombres) de edades comprendidas entre los 15 y los 19 años, estudiantes de $4 .^{\circ}$ curso de Educación Secundaria Obligatoria y de $2 .^{\circ}$ de Bachillerato, de los IES Bachiller Sabuco (Ciudad de Albacete) y Doctor Alarcón Santón (La Roda, Albacete). Del conjunto de estudiantes, 107 cursa el Bachillerato (71 estudia Física y/o Química) y 74 la ESO (39 estudia Física y Química). A partir de ahora, a los estudiantes que cursan Física y/o Química les llamaremos estudiantes de ciencias, y al resto los denominaremos estudiantes de letras.

Estos sujetos no parecen tener, a priori, características especiales que los diferencien de otros grupos de los respectivos cursos. No obstante, hemos de indicar que no se realizó muestreo aleatorio alguno, ya que se trató de una muestra de conveniencia. Por ello, los resultados no pueden ser extrapolados a toda la población estudiantil de $2 .^{\circ}$ de Bachillerato y $4 .^{\circ}$ de ESO, esto es, no hay garantías de validez externa.

\subsection{Materiales}

Teniendo presente que entran en juego en esta investigación la complejidad estructural de los problemas y la familiaridad de su contexto, hemos elaborado dos tipos de cuadernillos diferentes (Anexo 1), que tienen en común un pequeño formulario sobre la trayectoria y características personales del estudiante y las instrucciones para su cumplimentación. Ambos cuadernillos tienen dos problemas (Problema 1 y Problema 2) que difieren en su complejidad estructural. Ambos problemas tienen una estructura de proporcionalidad directa entre las variables y se resuelven mediante reglas de tres simples directas. La diferencia entre el primer y segundo problema radica en el número de reglas de tres simples directas que se han de llevar a cabo. En el caso del primer problema, solamente se ha de hacer una; en cambio, en el segundo hay que hacer dos (como mínimo), ligando la segunda con la primera. Por consiguiente, el primer problema tiene una complejidad estructural menor que el segundo. Así, el primero podemos calificarlo de «estructura sencilla o fácil» y el segundo de «estructura compleja o difícil». Por otra parte, la diferencia entre los dos tipos de cuadernillos está en el contexto de los 
problemas que incluyen (que no en la estructura, ya que hemos cuidado que la estructura de los problemas de cada contexto fuera, literalmente, simétrica). Uno de los contextos hace referencia al consumo de combustible de un coche (variables intervinientes en el primer problema: volumen de combustible y distancia recorrida; en el segundo problema: volumen de combustible, distancia recorrida y velocidad). El otro contexto está relacionado con la producción de un compuesto químico, la luteína (variables intervinientes en el primer problema: masa de luteína y fábricas en las que se produce; en el segundo problema: masa de luteína, fábricas y países donde se encuentran las fábricas).

Los dos tipos de cuadernillos tienen, además de los dos problemas, una pregunta en la que se solicita al estudiante que se defina acerca de si los problemas que ha resuelto son similares o diferentes, y razone el porqué de su respuesta. Con esta pregunta pretendemos conocer si el estudiante ha sido capaz de acceder o captar la estructura de los problemas. Es decir, una respuesta correcta a esta pregunta supondría afirmar que, por una parte, son similares, ya que ambos problemas se resuelven mediante reglas de tres simples directas (proporcionalidad directa entre las variables) y tienen el mismo contexto; y por otra parte son diferentes, dado que el número de reglas de tres simples directas que se han de llevar a cabo es distinto (diferente estructura).

También hay en ambos tipos de cuadernillos una pregunta de opción múltiple en la que se pide al estudiante que escoja hasta dos estrategias que haya utilizado en la resolución de los problemas. Las opciones ofrecidas en esta pregunta están extraídas de un estudio realizado por Solaz-Portolés, Rodríguez, Gómez y Sanjosé (2010), a las cuales se ha añadido una nueva opción que consideramos que puede tener cierta relevancia (esta opción plantea la posibilidad de aplicación mecánica de leyes, fórmulas o ecuaciones para resolver un problema). Con esta pregunta de opción múltiple se busca averiguar si el estudiante selecciona la opción que describe una estrategia de encontrar similaridades con otros problemas resueltos anteriormente, establecer las oportunas correspondencias y transferir ideas, esto es, si ha empleado la analogía (o la transferencia analógica) como estrategia de resolución.

\subsection{Procedimiento}

La administración de las pruebas se llevó a cabo en una sesión de clase. A los estudiantes participantes en este estudio se les informó previamente de la importancia que tendría la calificación obtenida en las pruebas para conocer su nivel en resolución de problemas y qué habilidades y conocimientos tienen en este campo. A continuación se distribuyeron al azar ambos tipos de cuadernillos. Dispusieron de un tiempo máximo de 50 minutos.

Para la calificación de los problemas se aplicó un protocolo de corrección elaborado por los autores de este trabajo y revisado por un profesor universitario ajeno a esta investigación (Anexo 2). La calificación máxima para cada problema 
es de 10 puntos. En la cuantificación de la pregunta relacionada con la estructura de los problemas se llevó a cabo previamente una primera lectura de las respuestas y la correspondiente categorización. Se decidió otorgar 2 puntos si el estudiante establece claramente las similaridades y las diferencias de los problemas que han intentado resolver (capta totalmente la estructura), 1 punto si lo hace parcialmente (capta parcialmente la estructura) y 0 puntos si no lo hace (no capta la estructura). Tanto la evaluación de la pregunta sobre la captación de la estructura de los problemas como de la resolución de los problemas se realizó entre un autor de este trabajo y un profesor universitario. En el primer caso, el valor del índice kappa de Cohen fue de 0,61 (error estándar 0,07, intervalo de confianza del 95\% comprendido entre los valores 0,47 y 0,76); en el segundo caso, el valor de kappa fue de 0,80 (error estándar 0,07, intervalo de confianza del 95\% comprendido entre los valores 0,65 y 0,94$)$. Estos valores de kappa nos indican un grado de acuerdo entre jueces bueno.

Finalmente en la pregunta de opción múltiple únicamente computamos si el estudiante selecciona, o no, la opción relacionada con el uso de analogías en la resolución de los problemas: 1 punto si la selecciona, 0 puntos si no lo hace.

\section{RESUlTAdos}

En la Tabla 1 se recogen las puntuaciones promedio de los estudiantes en los problemas 1 y 2 en función del nivel académico (curso) e itinerario cursado, del tipo de problema resuelto y de la captación de la estructura de los problemas. Se proporciona también el número de estudiantes en cada caso. En la Tabla 2 se ofrece la misma información, pero se ha sustituido la variable "captación de la estructura de los problemas» por la variable «utilización de analogías durante la resolución de los problemas". 

EFECTOS DE LAS CARACTERÍSTICAS DEL PROBLEMA...

Tabla 1. Puntuación PROMEdio EN los PROBlemas (CON SU DESVIACión TíPiCa)

DE ACUERDO CON EL NIVEL ACADÉMICO E ITINERARIO, EL TIPO DE PROBLEMA

Y LA CAPTACIÓN DE LA ESTRUCTURA DE LOS PROBLEMAS. TAMBIÉN SE INCLUYE

EL NÚMERO DE SUJETOS EN CADA CASO

\begin{tabular}{|c|c|c|c|c|}
\hline $\begin{array}{c}\text { NIVEL ACADÉMICO } \\
\text { E ITINERARIO }\end{array}$ & $\begin{array}{c}\text { TIPO } \\
\text { DE PROBLEMA }\end{array}$ & $\begin{array}{l}\text { CAPTACIÓN } \\
\text { DE LA } \\
\text { ESTRUCTURA }\end{array}$ & $\begin{array}{c}\text { PUNTUACIÓN } \\
\text { DEL PROBLEMA (SD) }\end{array}$ & $\begin{array}{l}\text { NÚMERO } \\
\text { DE SUJETOS }\end{array}$ \\
\hline \multirow{6}{*}{$\begin{array}{l}4 .^{\circ} \mathrm{ESO} \\
\text { Ciencias }\end{array}$} & \multirow{3}{*}{ Problema 1} & No & $6,5(3,6)$ & 38 \\
\hline & & Parcial & $10(0,0)$ & 1 \\
\hline & & Sí & - & 0 \\
\hline & \multirow{3}{*}{ Problema 2} & $\mathrm{No}$ & $3,9(3,8)$ & 38 \\
\hline & & Parcial & $4,0(0,0)$ & 1 \\
\hline & & Sí & - & 0 \\
\hline \multirow{6}{*}{$\begin{array}{l}4 .^{\circ} \mathrm{ESO} \\
\text { Letras }\end{array}$} & \multirow{3}{*}{ Problema 1} & No & $3,5(2,9)$ & 34 \\
\hline & & Parcial & $8,0(0,0)$ & 1 \\
\hline & & Sí & - & 0 \\
\hline & \multirow{3}{*}{ Problema 2} & $\mathrm{No}$ & $1,8(2,2)$ & 34 \\
\hline & & Parcial & $2,0(0,0)$ & 1 \\
\hline & & Sí & - & 0 \\
\hline \multirow{6}{*}{$\begin{array}{l}\text { 2. } \\
\text { Bachillerato } \\
\text { Ciencias }\end{array}$} & \multirow{3}{*}{ Problema 1} & $\mathrm{No}$ & $8,0(3,0)$ & 58 \\
\hline & & Parcial & $9,6(3,5)$ & 5 \\
\hline & & Sí & $7,7(0,3)$ & 8 \\
\hline & \multirow{3}{*}{ Problema 2} & $\mathrm{No}$ & $7,4(3,5)$ & 58 \\
\hline & & Parcial & $8,0(0,4)$ & 5 \\
\hline & & Sí & $7,9(2,4)$ & 8 \\
\hline \multirow{6}{*}{$\begin{array}{l}\text { 2. }{ }^{\circ} \text { Bachillerato } \\
\text { Letras }\end{array}$} & \multirow{3}{*}{ Problema 1} & $\mathrm{No}$ & $4,9(3,8)$ & 34 \\
\hline & & Parcial & $8,5(2,0)$ & 2 \\
\hline & & Sí & - & 0 \\
\hline & \multirow{3}{*}{ Problema 2} & No & $1,7(3,4)$ & 34 \\
\hline & & Parcial & $0,5(0,7)$ & 2 \\
\hline & & Sí & - & 0 \\
\hline
\end{tabular}


PEDRO GARCÍA GALLEGO, VICENTE SANJOSÉ LÓPEZ Y JOAN J. SOLAZ-PORTOLÉS EFECTOS DE LAS CARACTERÍSTICAS DEL PROBLEMA...

TABla 2. Puntuación PROMEdio en los PROBlemas (CON SU DESVIACión TÍPICA) DE ACUERDO CON EL NIVEL ACADÉMICO E ITINERARIO, EL TIPO DE PROBLEMA Y LA UTILIZACIÓN DE ANALOGÍAS EN LA RESOLUCIÓN DE LOS PROBLEMAS.

TAMBIÉN SE INCLUYE EL NÚMERO DE SUJETOS EN CADA CASO

\begin{tabular}{|c|c|c|c|c|}
\hline $\begin{array}{c}\text { NIVEL ACADÉMICO } \\
\text { E ITINERARIO } \\
\end{array}$ & $\begin{array}{c}\text { TIPO } \\
\text { DE PROBLEMA }\end{array}$ & $\begin{array}{c}\text { UTILIZACIÓN } \\
\text { DE ANALOGÍAS }\end{array}$ & $\begin{array}{c}\text { PUNTUACIÓN } \\
\text { DEL PROBLEMA (SD) }\end{array}$ & $\begin{array}{l}\text { NÚMERO } \\
\text { DE SUJETOS }\end{array}$ \\
\hline \multirow{4}{*}{$\begin{array}{l}\text { 4. }{ }^{\circ} \text { ESO } \\
\text { Ciencias }\end{array}$} & \multirow{2}{*}{ Problema 1} & $\mathrm{No}$ & $6,4(3,7)$ & 29 \\
\hline & & Sí & $7,2(3,0)$ & 10 \\
\hline & \multirow{2}{*}{ Problema 2} & $\mathrm{No}$ & $4,8(4,3)$ & 29 \\
\hline & & Sí & $4,5(2,5)$ & 10 \\
\hline \multirow{4}{*}{$\begin{array}{l}4 .^{\circ} \text { ESO } \\
\text { Letras }\end{array}$} & \multirow{2}{*}{ Problema 1} & No & $3,6(2,7)$ & 27 \\
\hline & & Sí & $1,6(2,8)$ & 8 \\
\hline & \multirow{2}{*}{ Problema 2} & $\mathrm{No}$ & $1,9(2,8)$ & 27 \\
\hline & & Sí & $1,6(0,7)$ & 8 \\
\hline \multirow{4}{*}{$\begin{array}{l}\text { 2. }{ }^{\circ} \text { Bachillerato } \\
\text { Ciencias }\end{array}$} & \multirow{2}{*}{ Problema 1} & $\mathrm{No}$ & $8,2(2,3)$ & 54 \\
\hline & & Sí & $7,6(3,2)$ & 17 \\
\hline & \multirow{2}{*}{ Problema 2} & No & $7,3(3,5)$ & 54 \\
\hline & & Sí & $8,1(1,9)$ & 17 \\
\hline \multirow{4}{*}{$\begin{array}{c}\text { 2. }{ }^{\circ} \text { Bachillerato } \\
\text { Letras }\end{array}$} & \multirow{2}{*}{ Problema 1} & $\mathrm{No}$ & $4,8(3,8)$ & 29 \\
\hline & & Sí & $5,3(3,7)$ & 7 \\
\hline & \multirow{2}{*}{ Problema 2} & $\mathrm{No}$ & $1,7(3,4)$ & 29 \\
\hline & & Sí & $1,6(2,6)$ & 7 \\
\hline
\end{tabular}

Sendas pruebas Kolmogorov-Smirnov mostraron que las puntuaciones para el Problema 1 (P1) y para el Problema 2 (P2) no se ajustaron a distribuciones normales ( $p<, 001$ en ambos casos). La inspección de la distribución de puntuaciones en ambos problemas muestra que hubo pocos sujetos con puntuaciones intermedias (entre 3,0 y 6,0) y que las frecuencias se acumularon en las zonas extremas de las puntuaciones, bien la inferior (entre 0,0 y 2,0 puntos), bien la superior (entre 7,0 y 10 puntos). Los porcentajes acumulados entre 0,0 y 2,0 puntos fueron del $38 \%$ en P1 y del 58\% en P2. Por su parte, los porcentajes acumulados entre 7,0 y 10 puntos fueron del $51 \%$ en P1 y del $45 \%$ en P2. Es destacable que la proporción de puntuación nula $(0,0)$ fue elevada en ambos problemas: el 19\% en P1 y el 38\% en P2. Como puede apreciarse en las tablas anteriores, y tal como se esperaba, el problema de complejidad estructural menor, P1, obtuvo una puntuación superior al problema de mayor complejidad estructural, P2 (Wilcoxon test: $Z=-4,601$; $\mathrm{p}<, 001)$.

La diferencia entre las puntuaciones de P1 y P2 (P1-P2) fue influida por el contexto de los problemas (un factor entre-sujetos), tal como mostró el test no paramétrico de Mann-Whitney para P1-P2 ( $U=3362,5 ; \mathrm{p}=, 029)$. Las puntuaciones de P1 y P2 fueron más diferentes en el contexto más familiar (consumo de combustible) que en el contexto menos familiar (fabricación del compuesto químico luteína). 
Las consecuencias de la interacción significativa entre las variables «complejidad del problema» y "contexto» pueden verse en la Figura 1.

Figura 1. Puntuación en CAda CONTEXTO (CONSUmo De COMBustible O COMPuesto

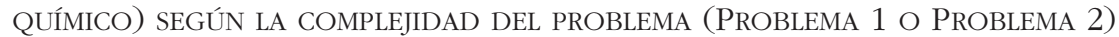
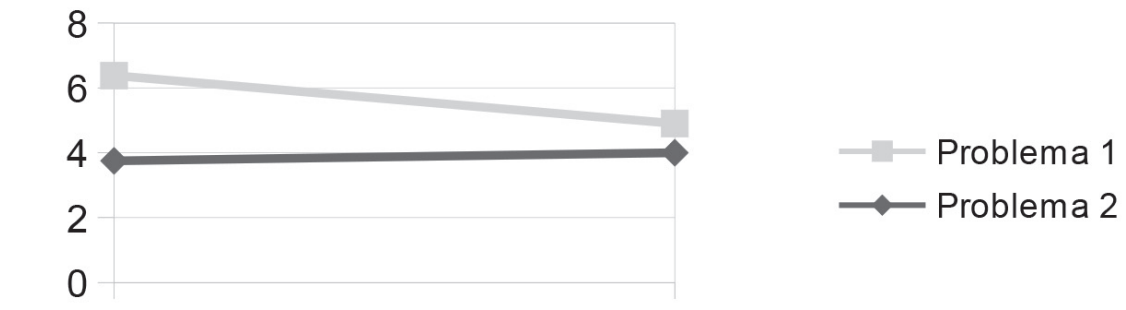

Cons. Comb.

Comp. Quím.

El nivel académico de los estudiantes ( $4 .^{\circ}$ ESO o $2 .^{\circ}$ Bachillerato) no resultó significativo sobre la diferencia P1-P2 ( $\mathrm{p}>$,30). Sin embargo, al considerar también el tipo de estudios cursados o itinerario (ciencias/letras) aparecieron efectos interesantes: en 4. ${ }^{\circ}$ de ESO los alumnos de letras y los de ciencias obtuvieron puntaciones similares en P1-P2 ( $\mathrm{p}>$,60), siendo siempre P2 más difícil que P1. Sin embargo,entre los estudiantes de $2^{\circ}$ de Bachillerato se produjo una diferencia sustancial entre los estudiantes de letras y los de ciencias en P1-P2. Para los primeros, P2 resultó claramente más difícil que P1, mientras que para los de ciencias ambos problemas resultaron de dificultad parecida (Mann-Whitney: $U=836,5 ; \mathrm{p}=, 001$ ). Éste es un efecto de la interacción significativa entre las tres variables "complejidad del problema", "nivel académico» e «itinerario cursado», y puede observarse en la Figura 2.

Figura 2. Puntuación en CADa itinerario y nivel aCAdÉmico ( $4 .^{\circ}$ ESO Ciencias, $4 .^{\circ}$ ESO Letras, $2 .^{\circ}$ BaChillerato Ciencias O $2 .^{\circ}$ BaChillerato Letras) EN FunCión de la Complejidad del PRoblema (Problema 1 o Problema 2)

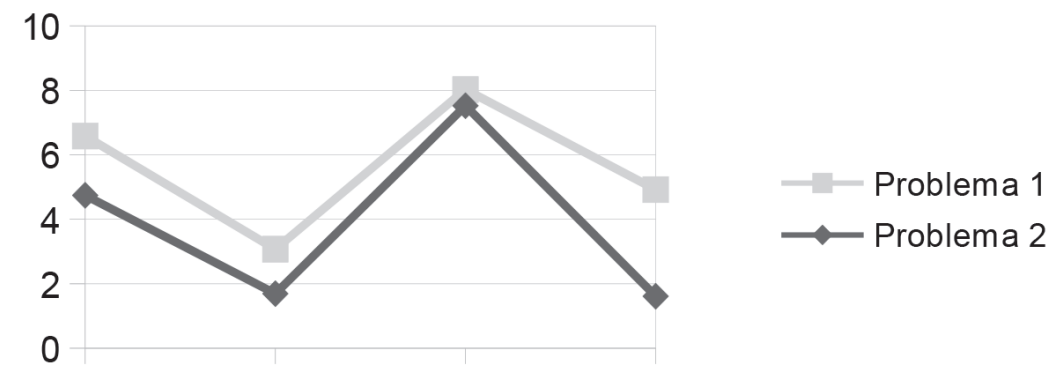

4ESO CC. 4ESO LL. 2BAC CC. 2BAC LL. 
Los estudiantes debían contestar cuestiones relacionadas con la estructura de los problemas y el uso de analogías durante la resolución. Nuestro interés se centró en manifestaciones acerca de las analogías y la percepción de las diferencias en complejidad estructural entre ambos problemas. Muy pocos estudiantes, 9,4\%, declararon similitudes y diferencias apropiadas entre los problemas, aunque el test de Mann-Whitney mostró diferencias significativas en las puntuaciones promedio de ambos problemas entre quienes no detectaron diferencias estructurales y quienes sí lo hicieron $(U=981,0 ; \mathrm{p}=, 043)$.

La probabilidad de no detectar diferencias apropiadas (en su complejidad estructural) entre P1 y P2 (usaremos ' $d=0$ ' para expresar esto), condicionada a obtener puntuación muy baja en P2 ('P2 = 0'), fue del 94\%. La misma probabilidad condicionada a que la puntuación en P1 fuera muy baja ('P1 = 0') llegó al 98\%. Esto parece indicar que la ausencia de detección de las diferencias en las estructuras de P1 y P2 estuvo fuertemente asociada al fracaso en su resolución o, dicho de otro modo, no detectar las diferencias apropiadas entre P1 y P2 fue condición (casi) suficiente para obtener muy bajas puntuaciones en P1 y en P2 (es decir: ' $\mathrm{d}=0 \rightarrow \mathrm{P} 1=0$ y P2 = 0' según la notación anterior). A la inversa, la probabilidad de obtener alta puntuación en P1 ('P1 = 1'), condicionada a haber detectado similitudes y diferencias apropiadas entre P1 y P2 (' $\mathrm{d}=1$ '), fue del 93\%, y la misma probabilidad condicionada para $\mathrm{P} 2$ fue del 67\%. Es decir, parece que el éxito en P1 estuvo asociado con haber comparado (o fue suficiente para comparar) de forma adecuada las estructuras de P1 y P2 (es decir: 'P1 = $1 \rightarrow \mathrm{d}=1$ '), aunque esa asociación no fue tan clara en el caso del éxito en P2. En un análisis complementario, muy bajas puntuaciones en P1 fueron condición suficiente para obtener muy bajas puntuaciones en $\mathrm{P} 2$ (' $\mathrm{P} 1=0 \rightarrow \mathrm{P} 2=0$ ' con probabilidad condicionada $=89 \%$ ), y muy altas puntuaciones en P2 fueron $\mathrm{P} 1=$ condición suficiente para obtener muy altas puntuaciones en $\mathrm{P} 1$ ('P2 $=1 \rightarrow \mathrm{P} 1=1$ ' con probabilidad condicionada $=92 \%$ ) o, lo que es lo mismo, obtener alta puntuación en P1 fue condición necesaria para obtener alta puntuación en P2, pero no al revés. Si se unen de forma lógica estas sentencias, $\mathrm{P} 1=1 \rightarrow \mathrm{d}=1$ y $\mathrm{P} 2=1 \rightarrow \mathrm{P} 1=1$, se concluye que $\mathrm{P} 2=1 \rightarrow \mathrm{d}=1$.

En cuanto al impacto del reconocimiento de analogías entre problemas, el hecho de que los estudiantes declarasen utilizar esta estrategia para resolver problemas o no lo hicieran no mostró significación alguna ( $\mathrm{p}>, 50$ en ambos problemas).

Las Figuras 3 y 4 nos ofrecen la puntuación en el Problema 1 y el Problema 2 según el nivel académico, el tipo de estudios de los sujetos y el contexto del problema (producción de compuesto químico o consumo combustible de un coche). 
Figura 3. Puntuación en el Problema 1 en función Del nivel aCADÉmiCo, ESTUDIOS CURSADOS Y CONTEXTO DEL PROBLEMA

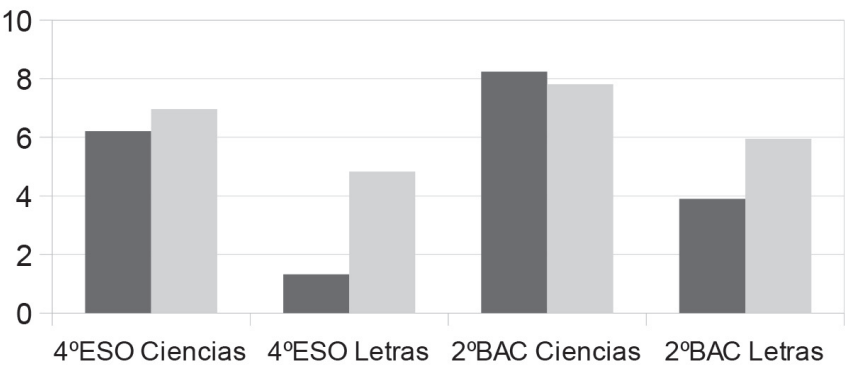

Comp. químico

Cons. combustible

Figura 4. Puntuación en el Problema 2 en función del nivel aCADÉmico, ESTUDIOS CURSADOS Y CONTEXTO DEL PROBLEMA

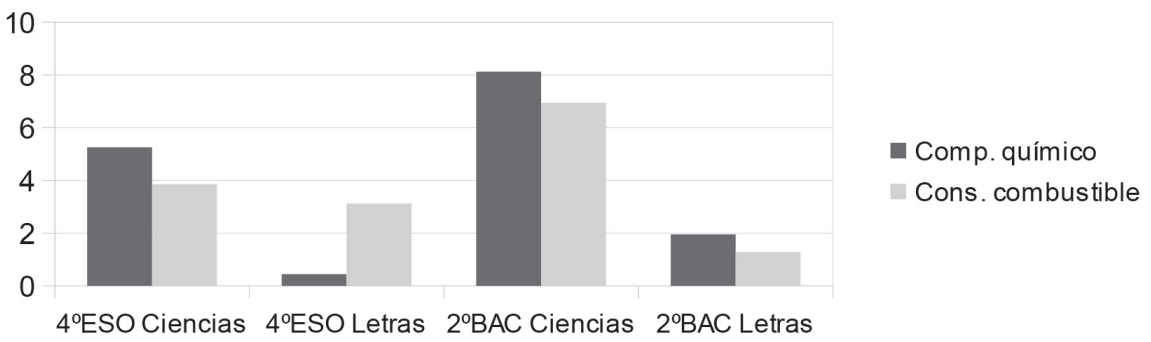

Las puntuaciones de los problemas se analizaron con sendos tests de MannWhitney. El nivel académico o curso resultó altamente significativo para ambos problemas (P1: $U=2880,0 ; \mathrm{p}=, 001 ; \mathrm{P} 2: U=2928,0 ; \mathrm{p}=, 002$ ): También el factor itinerario de los estudios (ciencias/ letras) resultó con alta significación, tanto en P1 $(U=1830,0 ; \mathrm{p}<, 001)$ como en P2 $(U=1544,5 ; \mathrm{p}<, 001)$. Para estudiar la importancia relativa de estos factores, se definieron los 4 subgrupos Nivel X Itinerario. Los rangos promedio se ordenaron del siguiente modo: $2 .^{\circ}$ Bach-Ciencias $>4 .^{\circ}$ ESO-Ciencias $>2 .^{\circ}$ Bach-Letras $>4 .^{\circ}$ ESO-Letras. Por tanto, el itinerario de los estudios resultó ser más importante que el nivel académico a la hora de resolver los problemas considerados en este estudio.

Con el fin de contrastar el efecto debido a la mayor o menor familiaridad del estudiante con el contexto del enunciado de los problemas, se realizaron también tests de Mann-Whitney, tomando como factor independiente el contexto de los problemas de cada cuadernillo, que fue una variable entre-sujetos del diseño. Este factor no fue significativo en P1 ( $p>, 15)$ ni tampoco en P2 ( $>$ > ,80). Sin embargo, análisis subsiguientes mostraron dos interacciones de interés entre este factor y otros que sí fueron significativas. Una de ellas ya la hemos 
mencionado, es la interacción entre complejidad estructural del problema y contexto. La otra es la interacción entre itinerario cursado y contexto. Así, los estudiantes de letras fueron más sensibles al factor contexto de los problemas (Mann-Whitney: $U=398,0$; $\mathrm{p}=, 006)$ que los de ciencias $(\mathrm{p}>, 29)$, como muestra la Figura 5.

FIgURA 5. PUNTUACIÓN PROMEDIO EN LOS PROBLEMAS EN FUNCIÓN DE LOS ESTUDIOS CURSADOS Y CONTEXTO DE LOS PROBLEMAS

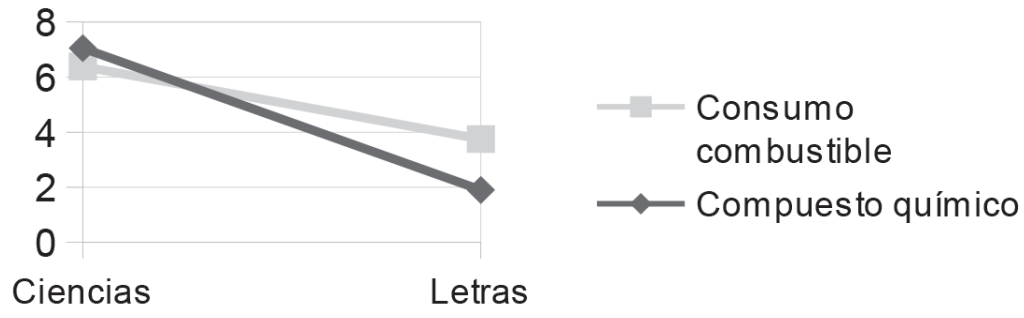

\section{DISCUSIÓN}

Se ha confirmado que a mayor complejidad estructural, mayor dificultad para resolverlo. Esto se ajusta plenamente a las bases psicológicas que explican la resolución de problemas (Goldstein, 2005). En concreto, se puede justificar teniendo en cuenta que un aumento de la complejidad estructural de un problema lleva asociado un aumento de la demanda cognitiva, que a su vez conduce a mayores dificultades para su resolución (Bogard, Lin y Chiang, 2013).

No obstante, hemos visto en este estudio que la variable complejidad estructural interacciona significativamente con otras, y es necesario resaltar dichas interacciones. En la primera interacción significativa, entre complejidad estructural y contexto del problema, se pone en evidencia que los problemas de estructura sencilla y contexto familiar para el estudiante resultan más fáciles de resolver, y que en contextos no familiares hay menos diferencia en el éxito de la resolución de problemas de distinta complejidad estructural, como puede verse en la Figura 1. En cuanto a la segunda, entre complejidad estructural, nivel académico e itinerario que cursa el estudiante, se traduce en que los estudiantes que cursan estudios de letras (humanidades y ciencias sociales) y tienen mayor nivel académico (2. ${ }^{\circ}$ de Bachillerato) se ven muy influidos en la resolución por la complejidad de la estructura de los problemas. En concreto, puntúan bastante menos en el problema más complejo. Por el contrario, los estudiantes de mayor nivel académico $\left(2{ }^{\circ}\right.$ de Bachillerato) y del itinerario de ciencias se ven muy poco afectados por la complejidad estructural (puntúan prácticamente igual en el problema de estructura simple y en el de compleja). Todo ello puede observarse en la Figura 2. 
De las pruebas estadísticas realizadas y de un análisis complementario parece concluirse que, en estudiantes de secundaria, la captación de la estructura del problema es muy importante para la resolución. Este resultado está totalmente de acuerdo con los presupuestos teóricos de la resolución de problemas, puesto que el acceso a la estructura de un problema es esencial para construir un modelo mental del problema, que permite abrir el camino hacia la resolución (Jonassen, 2003). No podemos dejar de indicar que se requeriría un estudio adicional específico, por ejemplo, mediante entrevistas clínicas, que fuera mucho más concluyente.

Sin embargo, el uso de analogías no ha mostrado asociación significativa con el desempeño en la resolución. Hemos de comentar en relación con esto que en este resultado obtenido puede haber influido claramente que nuestros estudiantes son todavía muy jóvenes para haber desarrollado conocimientos metacognitivos. Esto es, pensamos que no son todavía conocedores de las herramientas cognitivas y de los procesos cognitivos que utilizan (esto lo avanzamos a título de hipótesis). Por ello, no son capaces de saber si realmente utilizan o no analogías para resolver problemas, porque probablemente nunca se lo han planteado. En consecuencia, creemos que la aleatoriedad ha intervenido mucho en la elección de la opción en la pregunta en la que se solicita al estudiante qué estrategias ha utilizado durante la resolución de los problemas. A este respecto, señalaremos que expertos en resolución de problemas declaran que el uso de analogías es su principal estrategia en la resolución de problemas (Solaz-Portolés, Rodríguez, Gómez y Sanjosé, 2010).

En cuanto al papel de las variables nivel académico e itinerario cursado, de las Figuras 3 y 4 y de los correspondientes análisis estadísticos, se puede concluir que tanto el nivel académico como el tipo de estudios cursados son variables cruciales en el éxito en la resolución de problemas: a mayor nivel académico mayor éxito, y cursar estudios científicos mejora el desempeño en la resolución de los problemas. Además, hemos constatado en este trabajo la prevalencia del itinerario cursado sobre el nivel académico para resolver con éxito los problemas: los estudiantes que cursan ciencias resuelven mejor los problemas que hemos propuesto que los de letras, independientemente de su nivel académico. Esto resulta lógico si se atribuye a los estudiantes de ciencias las capacidades propias de un estilo cognitivo de independencia de campo. De hecho, este supuesto no resulta descabellado dado que se ha encontrado que los estudiantes de secundaria independientes de campo obtienen mejores resultados en las asignaturas de matemáticas y ciencias (López, Hederich y Camargo, 2012).

En relación a los efectos del contexto de los problemas, hemos de subrayar que dicha variable, por sí sola, no ha generado diferencias estadísticamente significativas en la resolución de problemas, aunque sí sus interacciones con otras variables. En concreto, la interacción entre contexto e itinerario académico (Figura 5), que refleja que los estudiantes de letras se ven más influidos que los de ciencias ante contextos no familiares, ha producido diferencias significativas. También ocurre con la interacción entre contexto y complejidad estructural, de la que ya se ha dado cumplida cuenta anteriormente (Figura 1). Estos resultados son coherentes 
con los obtenidos por Gómez-Ferragud, Solaz-Portolés y Sanjosé (2013a) y por Albarracín y Gorgorió (2013). Los primeros encontraron menor éxito en la detección de similaridad estructural (y, por consiguiente, menor probabilidad de transferencia analógica) entre problemas cuando éstos tienen un contexto poco familiar (relacionado con las ciencias) que cuando el contexto está relacionado con cuestiones más conocidas por los estudiantes. Los segundos descubrieron que el contexto de los problemas influye en las propuestas de resolución de los estudiantes.

\section{CONCLUSIONES E IMPLICACIONES DIDÁCTICAS}

En primer lugar, se ha comprobado la trascendencia de variable complejidad estructural de un problema sobre su resolución. En concreto, cuanto más compleja es la estructura del problema (en concreto, mayor número de variables y de relaciones matemáticas entre ellas), tanto mayor es la dificultad para resolverlo. Así pues parece confirmarse nuestra primera hipótesis. De ello, se deriva la necesidad didáctica de que el profesor evalúe la demanda cognitiva asociada con la complejidad estructural de los problemas y vaya ajustándolos a las capacidades de los estudiantes.

La hipótesis 2 defendía que el desempeño en la resolución de problemas debía estar asociado, además de a la captación de la estructura, al uso de analogías como estrategia de resolución. En nuestra investigación se ha visto que detectar diferencias estructurales entre problemas (esto es, captar su estructura) es imprescindible para tener éxito en su resolución. En cambio, el uso de analogías no ha mostrado asociación significativa con el desempeño en la resolución. En consecuencia, todo apunta a que la hipótesis 2 sólo ha quedado parcialmente confirmada. No obstante, pensamos que este resultado es meramente tentativo, sobre todo en lo que se refiere al uso de analogías, porque de la manera que se ha obtenido no es suficientemente precisa y se requería un estudio posterior.

Se ha podido observar que él éxito de los estudiantes de secundaria en la resolución de los problemas planteados viene condicionado por su nivel académico y por el itinerario cursado. Esto se ajusta plenamente a las previsiones que fueron recogidas en la hipótesis 3 que, de acuerdo con nuestros resultados, se verifica en todos sus términos.

Nuestra última hipótesis (hipótesis 4), que ponía de manifiesto el papel decisivo del contexto de los problemas para conseguir una resolución exitosa, no ha recibido apoyo experimental en este estudio. Eso sí, el contexto se ha revelado como una variable muy influyente en estudiantes con poca experiencia en resolución de problemas y en los problemas que resultan fáciles para los estudiantes (esto es, poco complejos estructuralmente). Esta circunstancia no debería ser pasada por alto por el profesorado de secundaria, que debería ser consciente de la necesidad de plantear problemas de contextos conocidos para el estudiante si quiere que puedan ser provechosos didácticamente. Dicho de otro modo, abordar 
la resolución de problemas sin tener una comprensión adecuada de los conceptos y situaciones que en ellos se plantean es un sinsentido pedagógico.

De la relevancia de la captación de la estructura en la resolución de los problemas creemos que se deriva la necesidad de instruir a nuestros estudiantes sobre el conocimiento esquemático o de esquemas de problema (Solaz-Portolés y Sanjosé, 2008b). Dicho conocimiento puede facilitar al estudiante la distinción entre el contexto y la estructura del problema, y la capacidad de acceder a la estructura. En este sentido, y dada la trascendencia que ha mostrado la combinación del contexto y la estructura de los problemas, creemos que sería muy aconsejable resolver bastantes problemas de la misma estructura y contextos variados, y al revés. El trabajo de Gomez-Ferragud, Solaz-Portolés y Sanjosé (2013b), llevado a cabo con diferentes sujetos, materiales y procedimientos, se decanta hacia las mismas ideas expuestas. Estos contenidos instruccionales se podrían incluir en actividades de carácter metacognitivo como parte del currículo. Tales actividades deberían tratar, entre otros aspectos, la existencia de diferentes tipos de conocimiento (declarativo, procedimental, situacional, etc.), la importancia del conocimiento esquemático de problemas, el uso de heurísticos en la resolución de problemas y la explicación del papel de las estrategias cognitivas y metacognitivas en los distintos pasos o procesos de resolución de problemas (aquí se tendría que analizar el papel de las analogías como estrategia de resolución de problemas).

Por último, no podemos dejar de señalar que la principal limitación de la presente investigación está relacionada con la naturaleza y el tamaño de la muestra. Los resultados y conclusiones que hemos obtenido sólo pueden ser válidos stricto sensu para los sujetos que han intervenido en ella. Otras limitaciones pueden tener su origen en los instrumentos que hemos empleado, en especial el utilizado para saber si los estudiantes han captado la estructura de los problemas y si han utilizado analogías para resolverlos. También cabe hacer referencia a aquellas variables que no hemos podido o sabido controlar y que pueden haber influido en los resultados. Por todo ello, creemos que este estudio debería replicarse con mayor número de estudiantes y niveles académicos, y también mediante instrumentos más precisos.

\section{REFERENCIAS BIBLIOGRÁFICAS}

Albarracín, Ll. y Gorgorió, N. (2013) Problemas de estimación de grandes cantidades: modelización e influencia del contexto. Revista Latinoamericana de Investigación en Matemática Educativa, 16 (3), 289-315. http://dx.doi.org/10.12802/relime.13.1631.

BARnetT, S. M. y CECI, S. J. (2002) When and where do we apply what we learn? A taxonomy for far transfer. Psychological Bulletin, 128, 612-637. http://dx.doi.org/10.1037/00332909.128.4.612.

Bogard, T.; LiU, M. y Chiang, V. (2013) Thresholds of Knowledge Development in Complex Problem Solving: A Multiple-Case Study of Advanced Learners' Cognitive Processes. Educational Technology Research and Development, 61 (3), 465-503. http://dx.doi. org/10.1007/s11423-013-9295-4. 
PEDRO GARCÍA GALLEGO, VICENTE SANJOSÉ LÓPEZ Y JOAN J. SOLAZ-PORTOLÉS EFECTOS DE LAS CARACTERÍSTICAS DEL PROBLEMA...

Chi, M. T. H.; Feltovich, P. J. y Glaser, R. (1981) Categorization and representation of physics problems by novices and experts. Cognitive Science, 5, 121-152. http://dx.doi. org/10.1207/s15516709cog0502_2.

De La Fuente, J. (1992) El problema de recordar un problema para resolver un problema. Anuario de Psicología, 52, 17-39.

FARIES, J. M. y ReISER, B. J. (1988) Access and use of previous solutions in a problem solving situation. Proceedings of the Tenth Annual Conference of the Cognitive Science Society. Montreal. Consultado el 13 de diciembre de 2013 http://www.dtic.mil/dtic/tr/fulltext/ u2/a224717.pdf.

Goldstein, E. B. (2005) Cognitive Psychology. Connecting Mind, Research, and Everyday Experience. Belmont, Thomson Wadsworth.

Goldstone, R. L. y SAKAMOTO, Y. (2003) The transfer of abstract principles governing complex adaptative systems. Cognitive Psychology, 46, 414-466. http://dx.doi.org/10.1016/ S0010-0285(02)00519-4.

Gómez-Ferragud, C. B.; Solaz-Portolés, J. J. y Sanjosé, V. (2013a) Analogy construction and success in mathematics and science problem-solving: a study with secondary students. Journal of Psychodidactics, 18 (1), 81-108.

Gómez-Ferragud, C. B.; Solaz-Portolés, J. J. y Sanjosé, V. (2013b) Efectos de la similitud superficial y estructural sobre la transferencia a partir de análogos en problemas de alta y baja familiaridad: primeros resultados. Enseñanza de las Ciencias, 31 (1), 135-151.

Gómez-Ferragud, C. B.; Solaz-Portolés, J. J. y SAnjosé, V. (2014) Dificultades para codificar, relacionar y categorizar problemas verbales algebraicos: dos estudios con estudiantes de secundaria y profesores en formación. Aceptado para su publicación en Bolema: Mathematics Education Bulletin. http://dx.doi.org/10.1387/RevPsicodidact. 5533.

Holland, J. H.; Holyoak, K. J.; Nisbett, R. E. y Thagard, P. (1986) Induction: Processes of Inference, Learning, and Discovery. Cambridge-MA, MIT Press.

HOLYOAK, K. J. (1984) Analogical thinking and human intelligence, en STERnBERG, R. J. (ed.) Advances in the psychology of human intelligence (vol. 2). Hilsdale-Nv, Erlbaum, 199-230.

HOLYOAK, K. J. y KOH, K. (1987) Surface and structural similarity in analogical transfer. Memory \& Cognition, 15 (4), 332-340. http://dx.doi.org/10.3758/BF03197035.

Jonassen, D. H. (2003) Using cognitive tools to represent problems. Journal of Research in Technology in Education, 35 (3), 362-381. http://dx.doi.org/10.1080/15391523.2003.1 0782391.

Keane, M. T. (1988) Analogical problem solving. Chichester, Ellis Horwood.

KINTSCH, W. (1998) Comprehension: a paradigm for cognition. Cambridge-UK, Cambridge University Press.

KintsCH, W. y GREENO, J. (1985) Understanding and solving word arithmetic problems. Psychological Review, 92 (1), 109-129. http://dx.doi.org/10.1037/0033-295X.92.1.109.

KuRTZ, K. J. y Loewestein, J. (2007) Converging on a new role for analogy in problem solving and retrieval: When two problems are better than one. Memory \& Cognition, 35 (2), 334-341. http://dx.doi.org/10.3758/BF03193454.

López, O.; Hederich, C. y Camargo, A. (2012) Logro en matemáticas, autorregulación del aprendizaje y estilo cognitivo. Suma Psicológica, 19 (2), 39-50.

Newel, A. y Simon, H. A. (1972) Human problem solving. Englewood Cliffs-NJ, Prentice Hall.

Rumelhart, D. E. (1989) Toward a microstructural account of human reasoning, en VosNiAdou, S. y Ortony, A. (eds.) Similarity and analogical reasoning. Cambridge, Cambridge University Press, 298-312. 
Sanjosé, V.; Solaz-Portolés, J. J. y Valenzuela, T. (2009) Transferencia inter-dominios en resolución de problemas: una propuesta instruccional basada en el proceso de "traducción algebraica». Enseñanza de las Ciencias, 27 (2), 169-184.

Solaz-Portolés, J. J.; Rodríguez, C.; Gómez, A. y Sanjosé, V. (2010) Conocimiento metacognitivo de las estrategias y habilidades mentales utilizadas para resolver problemas: un estudio con profesores de ciencias en formación. Didáctica de las Ciencias Experimentales y Sociales, 24, 139-152.

Solaz-Portolés, J. J. y SAnjosé, V. (2008a) Conocimientos y procesos cognitivos en la resolución de problemas de ciencias: consecuencias para la enseñanza. Magis. Revista Internacional de Investigación en Educación, 1 (1), 147-162.

SOlaz-PORTOlÉs, J. J. y SANJOSÉ, V. (2008b) Types of knowledge and their relations to problem solving in science: directions for practice. Sísifo. Educational Sciences Journal, 6, 105112. Consultado el 5 de noviembre de 2013 http://sisifo.fpce.ul.pt.

Solaz-Portolés, J. J.; SAnjosé, V. y Gangoso, Z. (2013) La investigación en resolución de problemas instruccionales. Efectos de variables del problema y de las variables cognitivas, metacognitivas y motivacionales del resolutor, en Benegas, J.; Pérez de Landazábal, M. C. y Otero, J. (eds.) El aprendizaje activo de la Física Básica Universitaria. Santiago de Compostela, Andavira Editora, 95-118.

SOlaz-Portolés, J. J.; SANjosé, V. y Gómez, C. (2011) La investigación sobre la influencia de las estrategias y la motivación en resolución de problemas: implicaciones para la enseñanza. Latin American Journal of Physics Education, 5 (4), 788-795.

SternberG, R. J. (1982) Reasoning, problem solving, and intelligence, en STERnBerG, R. J. (ed.) Handbook of human intelligence. Cambridge, Cambridge University Press, 225-307.

VAn LeHN, K. (1990) Problem solving and cognitive skill acquisition, en SimON, H. A. y Kaplan, C. A. (eds.) Foundations of Cognitive Science. Cambridge-ma, Harvard University Press, 1-52.

Verhoeven, L. y Perfetti, C. (2008) Advances in text comprehension: model, process and development. Applied Cognitive Psychology, 22, 293-301. http://dx.doi.org/10.1002/ acp.1417. 


\section{ANEXO 1 \\ CUADERNILLOS (TIPO 1 Y TIPO 2)}

NOMBRE Y APELLIDOS:

EDAD: SEXO:

Rodea la respuesta adecuada.

- ¿Cuál es tu nivel académico?: 4. ${ }^{\circ}$ ESO / 2. ${ }^{\circ}$ Bachiller

- ¿Cursas Física y/o Química?: SÍ / NO

Intenta resolver los dos problemas que tienes a continuación:

1) Un coche tiene un consumo medio de combustible de 6 litros cada 100 $\mathrm{km}(6 \mathrm{~L} / 100 \mathrm{~km})$. Si en dos tercios $(2 / 3)$ del trayecto total efectuado ha consumido 15 litros, ¿cuántos litros habrá consumido en todo el trayecto?

2) Un coche tiene un consumo medio de 6 litros cada $100 \mathrm{~km}(6 \mathrm{~L} / 100 \mathrm{~km})$ cuando circula a una velocidad de $100 \mathrm{~km} / \mathrm{h}$. Si dos tercios (2/3) del trayecto total los recorreenmedia(1/2)hora, ¿cuántoslitroshabrá consumidoentodoeltrayecto? A continuación te vamos a pedir información sobre estos problemas y sobre cómo los has intentado resolver.

I) Estos problemas te han parecido (señala lo que piensas y responde a la pregunta; si señalas ambas posibilidades, responde también a ambas):

1. Similares. ¿Por qué?

2. Diferentes. ¿Por qué?

II) Para resolverlos ¿cuál de estas estrategias has utilizado? (puedes señalar hasta dos posibilidades):

a) Dejo vagar mi mente libremente y trato de producir tantas ideas como pueda. Sólo después de haber producido muchas ideas, me pongo a analizar, juzgar y elegir las mejores.

b) Trato de recordar los problemas resueltos con éxito del pasado que son similares al problema planteado. Busco en dichos problemas los aspectos, elementos o características que se comparten con el problema planteado para transferir ideas que me puedan ser útiles. 
c) Trato de averiguar cuál es el principio, regla o ecuación que se adapta a los datos y a la pregunta. Luego la aplico sin necesidad de buscar otros problemas parecidos que ya conozco.

d) Trato de ir de forma sistemática a buscar la secuencia de pasos para llegar a la solución de manera gradual. De este modo, puedo identificar objetivos intermedios, planificar, programar y ordenar las operaciones que he de realizar.

e) Trato de representar el problema, es decir, representarlo en mi mente mediante imágenes. Intento ver la situación con mi imaginación, me hago dibujos, esquemas gráficos, etc.

f) Trato de combinar los diferentes aspectos del problema. Trato de asociar, tal vez al azar, algunos elementos del problema de modo que pueda llegar a algún resultado.

\section{NOMBRE Y APELLIDOS:}

EDAD: SEXO:

Rodea la respuesta adecuada.

- ¿Cuál es tu nivel académico?: 4. ${ }^{\circ}$ ESO / 2. ${ }^{\circ}$ Bachiller

- ¿Cursas Física y/o Química?: Sí / NO

Intenta resolver los dos problemas que tienes a continuación:

1) Una empresa química produce $35 \mathrm{~g}$ de luteína (un compuesto químico que se utiliza en distintos fármacos) por fábrica (35 g/fábrica). Si en dos tercios (2/3) de las fábricas se sintetizan 210 g de luteína, ¿cuántos gramos de luteína producen todas las fábricas de la empresa química?

2) Una empresa química multinacional produce $35 \mathrm{~g}$ de luteína (un compuesto químico que se utiliza en distintos fármacos) por fábrica (35 g/ fábrica) y tiene 3 fábricas en cada país (3 fábricas/país). Si dos tercios (2/3) de las fábricas están en 40 países, ¿cuántos gramos de luteína producen todas las fábricas de la multinacional química?

A continuación te vamos a pedir información sobre estos problemas y sobre cómo los has intentado resolver.

I) Estos problemas te han parecido (señala lo que piensas y responde a la pregunta; si señalas ambas posibilidades, responde también a ambas):

a) Similares. ¿Por qué? 
b) Diferentes. ¿Por qué?

II) Para resolverlos ¿cuál de estas estrategias has utilizado? (puedes señalar hasta dos posibilidades):

a) Dejo vagar mi mente libremente y trato de producir tantas ideas como pueda. Sólo después de haber producido muchas ideas, me pongo a analizar, juzgar y elegir las mejores.

b) Trato de recordar los problemas resueltos con éxito del pasado que son similares al problema planteado. Busco en dichos problemas los aspectos, elementos o características que se comparten con el problema planteado para transferir ideas que me puedan ser útiles.

c) Trato de averiguar cuál es el principio, regla o ecuación que se adapta a los datos y a la pregunta. Luego la aplico sin necesidad de buscar otros problemas parecidos que ya conozco.

d) Trato de ir de forma sistemática a buscar la secuencia de pasos para llegar a la solución de manera gradual. De este modo, puedo identificar objetivos intermedios, planificar, programar y ordenar las operaciones que he de realizar.

e) Trato de representar el problema, es decir, representarlo en mi mente mediante imágenes. Intento ver la situación con mi imaginación, me hago dibujos, esquemas gráficos, etc.

f) Trato de combinar los diferentes aspectos del problema. Trato de asociar, tal vez al azar, algunos elementos del problema de modo que pueda llegar a algún resultado. 


\section{ANEXO 2 \\ PROTOCOLO PARA LA CALIFICACIÓN \\ DE LOS PROBLEMAS 1 Y 2}

La puntuación máxima para cada problema es de 10 puntos, repartidos en los apartados que se describen a continuación. Las puntuaciones de cada apartado son acumulables.

1. Elabora un diagrama y/o un resumen de la información más relevante de la información que proporciona el enunciado como base para iniciar su resolución

- Presente 2 puntos

- Presente parcialmente 1

- Ausente 0 puntos

2. Plantea correctamente la relación o relaciones de proporcionalidad directa entre las variables implicadas en el problema

\section{- Presente 2 puntos}

- Ausente 0 puntos

3. Opera adecuadamente a partir de la relación o relaciones establecidas anteriormente

$$
\begin{aligned}
& \text { - Presente } 2 \text { puntos } \\
& \text { - Ausente } 0 \text { puntos }
\end{aligned}
$$

4. Despeja correctamente la variable incógnita

$$
\text { - Presente } 2 \text { puntos }
$$

- Ausente 0 puntos

5. Presenta claramente la respuesta a la pregunta formulada en el enunciado con las apropiadas unidades

- Presente 2 puntos

- Ausente 0 puntos 